PROCEEDINGS OF THE

AMERICAN MATHEMATICAL SOCIETY

Volume 130, Number 4, Pages 975-985

S 0002-9939(01)06251-7

Article electronically published on October 12, 2001

\title{
ON THE SECANT VARIETIES TO THE TANGENTIAL VARIETIES OF A VERONESEAN
}

\author{
M. V. CATALISANO, A. V. GERAMITA, AND A. GIMIGLIANO \\ (Communicated by Michael Stillman)
}

\begin{abstract}
We study the dimensions of the higher secant varieties to the tangent varieties of Veronese varieties. Our approach, generalizing that of Terracini, concerns 0-dimensional schemes which are the union of second infinitesimal neighbourhoods of generic points, each intersected with a generic double line.

We find the deficient secant line varieties for all the Veroneseans and all the deficient higher secant varieties for the quadratic Veroneseans. We conjecture that these are the only deficient secant varieties in this family and prove this up to secant projective 4-spaces.
\end{abstract}

\section{INTRODUCTION}

The outstanding work by Zak [Z] has rekindled interest in the classical study of higher secant varieties to projective varieties (see e.g. [K], CJ]). In this paper we study the secant varieties of tangent varieties to Veronesean varieties.

The Veronesean varieties $V_{n, j}, n \geq 1, j \geq 2$ (e.g. see [Hr]), are the embeddings of $\mathbb{P}^{n}$ into $\mathbb{P}^{N}, N=\left(\begin{array}{c}j+n \\ n\end{array}\right)-1$, via the complete linear system $S_{j}$, where $S=$ $k\left[x_{0}, \ldots, x_{n}\right]$. Some of them (the first is actually the Veronese surface) have secant varieties which "do not have the expected dimension". E.g. the closure of the union of the secant lines to the Veronese surface $V_{2,2} \subset \mathbb{P}^{5}$ should, by a dimension count, fill up $\mathbb{P}^{5}$, while the dimension of the secant variety is actually 4 .

For a variety $V \subseteq \mathbb{P}^{N}$, let $\mathcal{S}^{s-1}(V)$ be the variety which is the closure in $\mathbb{P}^{N}$ of $\bigcup_{P_{1}, \ldots, P_{s} \in V}\left\langle P_{1}, \ldots, P_{s}\right\rangle$. If $\operatorname{dim} V=d$, the "expected dimension" of $\mathcal{S}^{s-1}(V)$ is $s d+s-1$ whenever this value is $\leq N$, otherwise we expect $\mathcal{S}^{s-1}(V)=\mathbb{P}^{N}$.

The "exceptional behaviour" of the dimension of secant varieties can be found among other varieties $\mathcal{S}^{s-1}\left(V_{n, j}\right)$. The problem of determining which of them has a "defective" dimension is related (via the theory of inverse systems or, equivalently, apolarity; e.g. see [IK], $[\mathrm{Ge}]$ ) to determining the Hilbert Function of a 0-dimensional scheme $X \subset \mathbb{P}^{n}$ made by the first infinitesimal neighbourhoods of $s$ generic points (this is equivalent to what is classically known as Terracini's Lemma).

Received by the editors February 25, 2000 and, in revised form, October 26, 2000.

2000 Mathematics Subject Classification. Primary 14N15; Secondary 14M12.

The first author was supported in part by MURST funds.

The second author was supported in part by MURST funds, and by the Natural Sciences and Engineering Research Council of Canada.

The third author was supported in part by the University of Bologna, funds for selected research topics, and by the P.R.R.N.I. "Geometria Algebrica e Algebra Commutativa".

(C)2001 American Mathematical Society 
In this paper we look for other varieties that have this kind of peculiarity with respect to the dimension of their secant varieties. We investigate the tangential varieties $T_{n, j}$ of the Veronesean varieties $V_{n, j}$ and relate the dimension of their $s$ secant varieties with the Hilbert Function of certain 0 -dimensional schemes $Z \subset \mathbb{P}^{n}$, supported at $s$ generic points and whose structure is given by the intersection of their second infinitesimal neighbourhood with a double line (in a sense this is a generalization of Terracini's Lemma). More precisely, we will prove that

$$
\operatorname{dim} \mathcal{S}^{s-1}\left(T_{n, j}\right)=\operatorname{dim}_{k}\left(L_{1}^{j-1}, \ldots, L_{s}^{j-1}, L_{1}^{j-2} M_{1}, \ldots, L_{s}^{j-2} M_{s}\right)_{j}-1=H(Z, j)-1
$$

where $L_{1}, \ldots, L_{s}, M_{1}, \ldots, M_{s}$ are $2 s$ generic linear forms in $k\left[x_{0}, \ldots, x_{n}\right]$.

In the first two sections we introduce the topics and describe the relationship between the secant varieties we want to study and the 0-dimensional schemes $Z$.

In the third section we determine several cases in which our secant varieties do not have the expected dimension; we describe completely what happens for $s=2$, i.e. for the varieties of secant lines, and for $j=2$ (tangential varieties to quadratic embeddings of $\mathbb{P}^{n}$ ).

The geometry of the exceptions that we have found in the third section lead us to conjecture that they are the only ones. The conjecture is stated in section 4 , where we prove it in several cases. A complete answer is achieved (see Proposition 4.6) for the varieties $\mathcal{S}^{s-1}\left(T_{n, j}\right)$ of secant $(s-1)$-spaces, when $s \leq 5$.

\section{A FiRst LOOK AT TANGENTIAL VARIETIES}

We will always work over an algebraically closed field of characteristic zero. Denote by $T_{n, j}$ the "tangential variety" of $V_{n, j}$, i.e. the variety which is the closure in $\mathbb{P}^{N}$ of $\bigcup_{P \in V_{n, j}} T_{P}\left(V_{n, j}\right)$. We will study the varieties $\mathcal{S}^{s-1}\left(T_{n, j}\right)$. We consider the Veronesean $V_{n, j}$ as given by the embedding of $\left(\mathbb{P}^{n}\right)^{*}=\mathbb{P} S_{1}=\mathbb{P}\left\langle x_{0}, \ldots, x_{n}\right\rangle$ into $\mathbb{P}^{N}=\mathbb{P} S_{j}$ defined by $\nu_{j}: \mathbb{P} S_{1} \rightarrow \mathbb{P} S_{j}$, with $\nu_{j}(L)=L^{j}, \forall L \in S_{1}$. With this point of view it is not hard to determine $T_{L^{j}}\left(V_{n, j}\right)$, the tangent space to $V_{n, j}$ at the point $L^{j}$. We pass to the affine (so we view $\nu_{j}: S_{1} \rightarrow S_{j}$ ) and consider the differential map $d \nu_{j}: T_{L}\left(S_{1}\right) \rightarrow T_{L^{j}}\left(S_{j}\right)$. If we choose a direction through $L$ in $S_{1}$, say $L+\lambda M$, we get that the image of the corresponding tangent vector in $T_{L^{j}}\left(S_{j}\right)$ is given by

$$
\lim _{\lambda \rightarrow 0} \frac{d}{d \lambda}(L+\lambda M)^{j}=\lim _{\lambda \rightarrow 0} j(L+\lambda M)^{j-1} M=j L^{j-1} M .
$$

Then, since $V_{n, j}$ is smooth, we have an isomorphism $d \nu_{j}: S_{1} \rightarrow T_{L^{j}}\left(\nu_{j}\left(S_{1}\right)\right)$, given by $M \rightarrow L^{j-1} M$, where we view $T_{L^{j}}\left(\nu_{j}\left(S_{1}\right)\right)$ as $\left\{L^{j-1} M \mid M \in S_{1}\right\}$.

Now define (again we are looking at the affine situation) $\phi: S_{1} \times S_{1} \rightarrow S_{j}$ with $\phi(L, M)=L^{j-1} M \in T_{L^{j}}\left(\nu_{j}\left(S_{1}\right)\right)$. From what we have seen, we get that the closure of the image of $\phi$ is the affine cone over $T_{n, j}$. Hence, if we want to compute $\operatorname{dim} T_{n, j}$, it is enough to determine $\operatorname{rank} d \phi=\operatorname{dim}_{k}(\operatorname{im} d \phi)$ at a generic point $(L, M)$. Proceeding as before, we can consider a line $(L, M)+\lambda(A, B)$ through $(L, M)$ and its image $\phi((L, M)+\lambda(A, B))=(L+\lambda A)^{j-1}(M+\lambda B)$. Since

$$
\begin{aligned}
\lim _{\lambda \rightarrow 0} & \frac{d}{d \lambda}(L+\lambda A)^{j-1}(M+\lambda B) \\
\quad & =\lim _{\lambda \rightarrow 0}(L+\lambda A)^{j-1} B+(j-1)(L+\lambda A)^{j-2} A(M+\lambda B) \\
& =L^{j-1} B+(j-1) L^{j-2} A M
\end{aligned}
$$


we have, for generic $(L, M)$, that $d \phi(A, B)=L^{j-1} B+(j-1) L^{j-2} A M$. So im $d \phi=$ $\left\langle L^{j-1} S_{1}, L^{j-2} M S_{1}\right\rangle$. This has (affine) dimension $2 n+1$ (since only $L^{j-1} M$ is common to $L^{j-1} S_{1}$ and $\left.L^{j-2} M S_{1}\right)$.

From this we obtain the (classically well known) result that:

Proposition 1.1. For all $n, j \in \mathbb{N}$,

$$
\operatorname{dim} T_{n, j}=2 n ; \quad \operatorname{Sing} T_{n, j}=V_{n, j} .
$$

Proof. We have $\operatorname{dim} T_{n, j}=\operatorname{dim}_{k}(\operatorname{im} d \phi)-1=2 n\left(\right.$ where $\operatorname{dim}_{k}(\operatorname{im} d \phi)$ is calculated at a generic point).

Now $\operatorname{dim}_{k}(\operatorname{im} d \phi)=\operatorname{dim}_{k}\left\langle L^{j-1} S_{1}, L^{j-2} M S_{1}\right\rangle=2 n+1$, except if $L=M$ (in this case $\left.\operatorname{dim}_{k}(\operatorname{im} d \phi)=\operatorname{dim}_{k}\left\langle L^{j-1} S_{1}\right\rangle=n+1\right)$; that is, when $\phi(L, L)=L^{j} \in V_{n, j}$. Hence Sing $T_{n, j} \subseteq V_{n, j}$. Since it is well known (and easy to check using the parameterization of $V_{n, j}$ ) that $V_{n, j} \subseteq \operatorname{Sing} T_{n, j}$, we are done.

\section{INVERSE SYSTEMS AND SECANT VARIETIES}

Now we consider in detail the space $W=\left\langle L^{j-1} S_{1}, L^{j-2} M S_{1}\right\rangle \subset S_{j}$, which corresponds to the affine cone over $T_{L^{j-1} M}\left(T_{n, j}\right)$. Let $R=k\left[y_{0}, \ldots, y_{n}\right] \simeq S$, and consider the action of $R$ on $S$, defined by (see [IK], Ge for details) $y_{i} \circ x_{j}=$ $\left(\partial / \partial x_{i}\right)\left(x_{j}\right)$. We use the standard properties of differentiation to extend this action of $R_{1}$ on $S_{1}$ to $R_{i} \times S_{j} \rightarrow S_{j-i}$. Note that if $i=j$ the resulting map to $k$ is a perfect pairing.

If $I$ is a homogeneous ideal in $R$, we define the inverse system of $I$, denoted $I^{-1}$, as the $R$-submodule of $S$ consisting of all elements of $S$ annihilated by $I$ (note: $I^{-1}$ is not (necessarily) an ideal in $S$ ).

If $L, M$ are generic, we can choose coordinates so that $W=\left\langle x_{0}^{j-1} S_{1}, x_{0}^{j-2} x_{1} S_{1}\right\rangle$. Since $W \subset S_{j}$ we can consider the space $I_{j}=W^{\perp} \subset R_{j}$ (the "perp" with respect to the perfect pairing mentioned above). An easy computation shows that if $I=$ $\left(y_{1}, \ldots, y_{n}\right)^{3}+\left(y_{2}, \ldots, y_{n}\right)^{2}$, then $W=\left(I^{-1}\right)_{j}$.

Note that for $n \geq 2, I$ represents a scheme $Z \subseteq \mathbb{P}^{n}$ given by the intersection of the second infinitesimal neighbourhood of the point $[1: 0: \ldots: 0]$ with the first infinitesimal neighbourhood of the line $l=\left\langle y_{0}, y_{1}\right\rangle$ (for $n=1$ we have $I=\left(y_{1}^{3}\right)$ instead).

Definition. A $(2,3)$-point in $\mathbb{P}^{n}$ is a 0 -dimensional scheme in $\mathbb{P}^{n}$ with support at one point $P$, and whose ideal is of type $\mathfrak{p}^{3}+I_{l}^{2}$, where $l \subset \mathbb{P}^{n}$ is a line through $P$ with defining ideal $I_{l}$ and $\mathfrak{p}$ is the ideal of $P$.

Since $\operatorname{dim}_{k} W+\operatorname{dim}_{k} I_{j}=\operatorname{dim}_{k} R_{j}=\left(\begin{array}{c}n+j \\ n\end{array}\right)$, we get $\operatorname{dim}_{k} W=H(Z, j)$, where $H(Z,$.$) is the Hilbert function of Z$. Thus, $Z$ is a degree $2 n+1$ structure on the point $[1: 0: \ldots: 0]$.

If we want to consider $\mathcal{S}^{s-1}\left(T_{n, j}\right)$, which we will indicate more compactly by $\mathcal{S}_{n, j}^{s-1}$, we can study the map $\phi_{s}:\left(S_{1} \times S_{1}\right)^{s} \rightarrow \mathcal{S}_{n, j}^{s-1}$, where $\phi_{s}\left(L_{1}, M_{1}, \ldots, L_{s}, M_{s}\right)=$ $\left(L_{1}^{j-1} M_{1}+L_{2}^{j-1} M_{2}+\ldots+L_{s}^{j-1} M_{s}\right)$. Again, for a generic choice of $L_{1}, M_{1}, \ldots, L_{s}, M_{s}$, the dimension of im $d \phi_{s}$ will tell us the dimension of $\mathcal{S}_{n, j}^{s-1}$. is

Using the same procedure as above, we get that the (affine) space $W_{n, j}^{s}=\operatorname{im} d \phi_{s}$

$$
W_{n, j}^{s}=\left(L_{1}^{j-1}, \ldots, L_{s}^{j-1}, L_{1}^{j-2} M_{1}, \ldots, L_{s}^{j-2} M_{s}\right)_{j},
$$


which can be viewed as the degree $j$ part $I_{j}^{-1}$ of $I^{-1}$, where $I$ is the ideal of a scheme $Z$ which is the union of $s$ general (2,3)-points, i.e. $I=\mathfrak{q}_{1} \cap \ldots \cap \mathfrak{q}_{s}$, and each $\mathfrak{q}_{i}=\mathfrak{p}_{i}^{3}+I_{l_{i}}^{2}$, where the $\mathfrak{p}_{i}$ 's are the ideals of $s$ generic points $P_{i}$ in $\mathbb{P}^{n}$ and each line $l_{i}$ is generic among the lines passing through $P_{i}$.

Again $\operatorname{dim}_{k} W_{n, j}^{s}+\operatorname{dim}_{k} I_{j}=\left(\begin{array}{c}n+j \\ n\end{array}\right)$, and $\operatorname{dim}_{k} W_{n, j}^{s}=H(Z, j)$, so determining $\operatorname{dim} \mathcal{S}_{n, j}^{s-1}$ reduces to determining $H(Z, j)$.

The expected value for $H(Z, j)$ is $\min \left\{\left(\begin{array}{c}n+j \\ n\end{array}\right), s(2 n+1)\right\}$ (if all the $\mathfrak{q}_{i}$ impose independent conditions on hypersurfaces of degree $j$ ), so we expect this value for $\operatorname{dim}_{k} W_{n, j}^{s}$. This gives the following expected dimension for $\operatorname{dim} \mathcal{S}_{n, j}^{s-1} \subset \mathbb{P}^{N}$ :

$$
\operatorname{dim} \mathcal{S}_{n, j}^{s-1}= \begin{cases}N & \text { if }\left(\begin{array}{c}
n+j \\
n
\end{array}\right) \leq s(2 n+1), \\
s(2 n+1)-1 & \text { if }\left(\begin{array}{c}
n+j \\
n
\end{array}\right) \geq s(2 n+1) .\end{cases}
$$

This is (of course) also what we expect by geometric intuition: $s(2 n+1)-1$ correspond to $\infty^{2 n s}$ choices of $s$ points on $T_{n, j}$, plus $\infty^{s-1}$ choices of a point on the $\mathbb{P}^{s-1}$ spanned by the $s$ points. When this number is too big, we expect $\mathcal{S}_{n, j}^{s-1}=\mathbb{P}^{N}$.

\section{THE EXCEPTIONS}

We look for cases where $Z$ fails to impose independent conditions on hypersurfaces of degree $j$.

3.1. The case $n=1$. In this case there are no exceptions, i.e.

Proposition 3.1. All the tangential varieties of rational normal curves $V_{1, j} \subset \mathbb{P}^{j}$ have secant varieties of the expected dimension, i.e. $\operatorname{dim} \mathcal{S}_{1, j}^{s-1}=\min \{3 s-1, j\}$, $\forall s, j \geq 1$.

Proof. In this case $W_{1, j}^{s}=\left(L_{1}^{j-1}, \ldots, L_{s}^{j-1}, L_{1}^{j-2} M_{1}, \ldots, L_{s}^{j-2} M_{s}\right)_{j}=\left(I^{-1}\right)_{j}$, where $I=\left(w_{1}^{3}\right) \cap\left(w_{2}^{3}\right) \cap \ldots \cap\left(w_{s}^{3}\right)=\left(w_{1}^{3} \cdot w_{2}^{3} \cdots w_{s}^{3}\right)$ and $w_{i}$ is the form in $R=k\left[y_{0}, y_{1}\right]$ corresponding to $M_{i}$. Then it is immediate to check that

$$
\operatorname{dim}_{k} W_{1, j}^{s}=j+1-\operatorname{dim}_{k} I_{j}= \begin{cases}j+1 & \text { if } j \leq 3 s-1 \\ 3 s & \text { if } j \geq 3 s-1\end{cases}
$$

and the conclusion follows.

3.2. The case $s=2$. This case is geometrically interesting since it is, in a sense, the "simplest". So, in this case, we are considering the varieties $\mathcal{S}_{n, j}^{1}$ of all the secant lines to $T_{n, j}$ (notice that we obviously have $\mathcal{S}_{n, j}^{1} \subseteq \mathcal{S}_{n, j}^{s-1}, \forall s \geq 2$ ).

Let us first consider $n=2$ (since $n=1$ has been checked).

For $j=2$ (i.e. for the tangential variety of the Veronese surface) we get $W_{2,2}^{2}=$ $\left(L_{1}, L_{2}, M_{1}, M_{2}\right)_{2}=S_{2}$, hence $\operatorname{dim}_{k} W_{2,2}^{2}=\operatorname{dim}_{k} S_{2}=6$ and $\operatorname{dim} \mathcal{S}_{2,2}^{1}=5$ (i.e. $\left.\mathcal{S}_{2,2}^{1}=\mathbb{P}^{5}\right)$, and the dimension is the expected one.

For $j=3$, things get more interesting. In fact, $W_{2,3}^{2}=\left(L_{1}^{2}, L_{2}^{2}, L_{1} M_{1}, L_{2} M_{2}\right)_{3}=$ $\left(I^{-1}\right)_{3}$, where $I=\mathfrak{q}_{1} \cap \mathfrak{q}_{2}$, which, for an appropriate choice of the coordinates, can be written as $\left(y_{0}^{3}, y_{0}^{2} y_{1}, y_{1}^{2}\right) \cap\left(y_{0}^{3}, y_{0}^{2} y_{2}, y_{2}^{2}\right)=\left(y_{0}^{3}, y_{0}^{2} y_{1} y_{2}, y_{0}^{2} y_{2}^{2}\right)$. Hence $\operatorname{dim}_{k} I_{3}=1$. This is not what was expected, since $\operatorname{deg} Z=10$ and $\operatorname{dim}_{k} R_{3}=10$, so one would expect that $I_{3}=0$. Consequently, we have $\operatorname{dim}_{k} W_{2,3}^{2}=\operatorname{dim}_{k} R_{3}-\operatorname{dim}_{k} I_{3}=9$, and so $\operatorname{dim} \mathcal{S}_{2,3}^{1}=8$, while one would expect that it would fill $\mathbb{P}^{9}$. 
For $j \geq 4$, things are different: $W_{2, j}^{2}=\left(I^{-1}\right)_{j}$, and $\operatorname{dim}_{k} I_{j}=\left(\begin{array}{c}j+2 \\ 2\end{array}\right)-10$, as it ought to be, since it is already so for $j=4\left(I_{4}=\left(y_{0}^{3}, y_{0}^{2} y_{1} y_{2}, y_{0}^{2} y_{2}^{2}\right)_{4}\right.$ is 5 dimensional). Hence $\operatorname{dim}_{k} W_{2, j}^{2}=\left(\begin{array}{c}j+2 \\ 2\end{array}\right)-\left(\begin{array}{c}j+2 \\ 2\end{array}\right)+10=10$ and $\operatorname{dim} \mathcal{S}_{2, j}^{1}=9, \forall j \geq 4$.

Now let $n \geq 3$. We have $W_{n, j}^{2}=\left(L_{1}^{j-1}, L_{2}^{j-1}, L_{1}^{j-2} M_{1}, L_{2}^{j-2} M_{2}\right)_{j}=\left(I^{-1}\right)_{3}$, and, by choosing appropriate coordinates, we can suppose

$$
I=\left(\left(y_{1}, y_{2}, \ldots, y_{n}\right)^{3}+\left(y_{2}, y_{3}, \ldots, y_{n}\right)^{2}\right) \cap\left(\left(y_{0}, y_{1}, y_{3}, \ldots, y_{n}\right)^{3}+\left(y_{0}, y_{1}, y_{4}, \ldots, y_{n}\right)^{2}\right) .
$$

Hence we can easily check that

$$
I_{2}=\left[\left(y_{4}, y_{5}, \ldots, y_{n}\right)^{2}\right]_{2},
$$

while

$$
\begin{aligned}
I_{3}=\left[\left(y_{4}, \ldots, y_{n}\right)^{2}+\left(y_{1}, y_{3}\right)^{3}+\left(y_{0} y_{3}^{2}, y_{1}^{2} y_{2}\right)\right. \\
\left.+\left(y_{1}^{2}, y_{3}^{2}, y_{0} y_{2}, y_{0} y_{3}, y_{1} y_{2}, y_{1} y_{3}\right)\left(y_{4}, \ldots, y_{n}\right)\right]_{3} .
\end{aligned}
$$

For $n=3$, this yields $\operatorname{dim}_{k} I_{2}=0, I_{3}=\left\langle y_{1}^{3}, y_{1}^{2} y_{3}, y_{1} y_{3}^{2}, y_{3}^{3}, y_{0} y_{3}^{2}, y_{1}^{2} y_{2}\right\rangle$, i.e. $\operatorname{dim}_{k} I_{3}=6$, which is the expected value.

When $n \geq 4, \operatorname{dim}_{k} I_{2}=\operatorname{dim}_{k}\left(\left(y_{4}, y_{5}, \ldots, y_{n}\right)^{2}\right)_{2}=\left(\begin{array}{c}n-2 \\ 2\end{array}\right)$, i.e., $H(Z, 2)=\left(\begin{array}{c}n+2 \\ 2\end{array}\right)-$ $\left(\begin{array}{c}n-2 \\ 2\end{array}\right)=4 n-2$, which is not the expected dimension (i.e. $\left.4 n+2\right) ; \operatorname{dim}_{k} I_{3}=$ $\left(\begin{array}{c}n-1 \\ 3\end{array}\right)+4\left(\begin{array}{c}n-2 \\ 2\end{array}\right)+4+2+6(n-3)$ and an easy computation shows that $H(Z, 3)=4 n+2$, as expected. This also implies that $H(Z, j)=4 n+2, \forall j \geq 4$.

We have proved the following proposition, which completely describes the case $s=2$ :

Proposition 3.2. For all $n, j, \operatorname{dim} \mathcal{S}_{n, j}^{1}=\min \left\{4 n+1,\left(\begin{array}{c}n+j \\ j\end{array}\right)-1\right\}$, as expected, with the following exceptions:

i) $\operatorname{dim} \mathcal{S}_{2,3}^{1}=8$;

ii) $\operatorname{dim} \mathcal{S}_{n, 2}^{1}=4 n-3, \forall n \geq 4$.

Remark. In case $i$ i) we have that $\operatorname{dim} \mathcal{S}_{n, 2}^{1}=4 n-3=\left(\begin{array}{c}n+2 \\ 2\end{array}\right)-1$ for $n=2,3$, while for $n \geq 4, \mathcal{S}_{n, 2}^{1}$ is defective. It is not hard to know what the geometry is in these cases. Consider the situation when $n=4$, i.e. two $(2,3)$-points, with ideals $\mathfrak{q}_{i}=\mathfrak{p}_{i}^{3}+I_{l_{i}}^{2}$. Now $l_{1} \cup l_{2}$ spans a hyperplane $H$ inside $\mathbb{P}^{4}$ and obviously $Z \subseteq 2 H$, even if $\operatorname{deg} Z=18$ should imply that $Z$ is not contained in any quadric.

3.3. The case $j=2$. The exceptions in case $i i$ ) of Proposition 3.2 can be generalized in quite a simple way: consider a 0 -dimensional scheme $Z$ made of $s$ generic (2,3)-points, with ideals of type $\mathfrak{q}_{i}=\mathfrak{p}_{i}^{3}+I_{l_{i}}^{2}, i=1, \ldots, s$, inside $\mathbb{P}^{n}$, $n \geq 2 s$. Let $n=2 s+k, k \geq 0$. We can choose coordinates $\left(y_{0}, \ldots, y_{2 s+k}\right)$ in $\mathbb{P}^{2 s+k}$ such that $\mathfrak{p}_{i}=\left(y_{0}, \ldots, \hat{y}_{2 i-2}, \ldots y_{2 s+k}\right)$ and $l_{i}=\left\langle y_{2 i-2}, y_{2 i-1}\right\rangle$ (i.e. $I_{l_{i}}=$ $\left.\left(y_{0}, \ldots, \hat{y}_{2 i-2}, \hat{y}_{2 i-1}, \ldots, y_{2 s+k}\right)\right)$.

Proceeding as before, we will get that $\left(I_{Z}\right)_{2}=\left(y_{2 s}, \ldots, y_{2 s+k}\right)^{2}$ and hence that $\operatorname{dim}_{k}\left(I_{Z}\right)_{2}=\left(\begin{array}{c}k+2 \\ 2\end{array}\right)$, while $\operatorname{deg} Z=s(2 n+1)=4 s^{2}+(2 k+1) s$. Thus, $\forall s \geq 2$,

$$
H(Z, 2)=\left(\begin{array}{c}
n+2 \\
2
\end{array}\right)-\left(\begin{array}{c}
k+2 \\
2
\end{array}\right)=s(2 n+1)-2\left(s^{2}-s\right)=\operatorname{deg} Z-2\left(s^{2}-s\right),
$$

as we just saw in Proposition 3.2 in case $s=2$, while the expected value for $H(Z, 2)$ is $\min \left\{\left(\begin{array}{c}n+2 \\ 2\end{array}\right), \operatorname{deg} Z\right\}$.

Notice also that if we have $n<2 s$, then $\left(I_{Z}\right)_{2}=0$. In fact, let $s^{\prime}<s$ be maximum such that $2 s^{\prime} \leq n$, i.e. $s^{\prime}=\frac{n}{2}$ or $s^{\prime}=\frac{n-1}{2}$. Then $Z^{\prime} \subseteq Z$, given by 
the first $s^{\prime}$ (multiple) points of $Z$, will be contained in either one or three quadrics $\left(\left(I_{Z^{\prime}}\right)_{2}=\left(y_{2 s^{\prime}}^{2}\right)_{2}\right.$ or $\left.I_{Z^{\prime}}=\left(\left(y_{2 s^{\prime}}, y_{2 s^{\prime}+1}\right)^{2}\right)_{2}\right)$, and it is immediate to see that the other $s-s^{\prime}$ multiple points which form $Z$ are not contained in those quadrics (since they are generic).

Hence we have proved the following (which completely describes the case $j=2$ ):

Proposition 3.3. $\forall s \geq 2$, we have that:

i) for $n<2 s, \mathcal{S}_{n, 2}^{s-1}=\mathbb{P}^{N}$, as expected;

ii) for $n \geq 2 s, \operatorname{dim} \mathcal{S}_{n, 2}^{s-1}=s(2 n+1)-2\left(s^{2}-s\right)-1$, i.e., it is $2\left(s^{2}-s\right)$ less than expected when $\left(\begin{array}{c}n+2 \\ 2\end{array}\right) \geq s(2 n+1)$, and it is $\left(\begin{array}{c}n-2 s+2 \\ 2\end{array}\right)$ less than expected for $\left(\begin{array}{c}n+2 \\ 2\end{array}\right) \leq s(2 n+1)$

3.4. Other cases. The exception we determined in Proposition $3.2 i$ ) can be generalized. We can consider schemes $Z$ which are the union of $n(2,3)$-points in $\mathbb{P}^{n}$. Clearly such a $Z$ is contained in the triple hyperplane passing through the simple points which are the support of $Z$. Since any $(2,3)$-point in $\mathbb{P}^{n}$ has degree $2 n+1$, we have that $\operatorname{deg} Z=2 n^{2}+n$. Since the space of degree 3 forms in $\mathbb{P}^{n}$ has dimension $\left(\begin{array}{c}n+3 \\ 3\end{array}\right)=\frac{n^{3}+6 n^{2}+11 n+6}{6}$, we see that whenever $\frac{n^{3}+6 n^{2}+11 n+6}{6} \leq 2 n^{2}+n$, then $H(Z, 3)$ is not what is expected (the triple hyperplane should not be there, and it is not hard to check, e.g. using CoCoA, that it is the only hypersurface of degree three through $Z$ ). An immediate computation shows that this happens for $2 \leq n \leq 4$, thus proving:

Proposition 3.4. $\operatorname{dim} \mathcal{S}_{3,3}^{2}=18$ (rather then 19), $\operatorname{dim} \mathcal{S}_{4,3}^{3}=33$ (rather than 34 ).

Note that a direct computation, using CoCoA, shows that when $Z$ is given by five $(2,3)$-points in $\mathbb{P}^{5}$, then $H(Z, 3)$ assumes its expected value (i.e. $H(Z, 3)=1$ ). Hence $\mathcal{S}_{5,3}^{4}$ has the expected dimension.

\section{A CONJeCture AND SOME EVIDENCE FOR IT}

Examples, and lack of other geometric reasons, lead us to conjecture that the cases where the dimension of $\mathcal{S}_{n, j}^{s-1}$ is not as expected are only those that we have found in the previous section. More precisely,

Conjecture 4.1. The secant variety $\mathcal{S}_{n, j}^{s-1}$ has the expected dimension, except when:

i) $j=2, n \geq 2 s$;

ii) $j=3, s=n=2,3,4$.

In the previous sections we proved the conjecture for $j=2$ and for $s=2$.

In what follows we are going to prove Conjecture 4.1 in some other cases. The main other tool that we will use in order to compute $H(Z, j)$ is "la methode d'Horace" (see [H] or [Gi] for an expository survey).

4.1. The case $n \geq s+1$. In this case, since we already studied the case $j=2$, we need only consider $j \geq 3$.

Proposition 4.2. Let $j \geq 3, n \geq s+1$. Then $\operatorname{dim} \mathcal{S}_{n, j}^{s-1}=s(2 n+1)-1$, i.e. the conjecture is true for $j \geq 3, n \geq s+1$.

Proof. We work by induction on $s$ : for $s=2$ the conclusion follows from Proposition 3.2 , so let $s>2$. It is enough to show that $H(Z, 3)=s(2 n+1)$ where $Z$ is made 
by $s(2<s \leq n-1)$ generic (2,3)-points (since this implies $H(Z, j)=s(2 n+1)$ $\forall j \geq 3)$.

Let $P_{1}, \ldots, P_{s}$ be the support of the $s(2,3)$-points, let $\mathfrak{p}_{i}$ be the ideal of $P_{i}$, and let $l_{i}=P_{i} Q_{i}\left(Q_{i} \neq P_{i}\right)$ be a generic line through $P_{i}$. We have $I=I_{Z}=$ $\left(\mathfrak{p}_{1}^{3}+I_{l_{1}}^{2}\right) \cap \ldots \cap\left(\mathfrak{p}_{s}^{3}+I_{l_{s}}^{2}\right)$. Let $J=J_{Z^{\prime}}=\left(\mathfrak{p}_{2}^{3}+I_{l_{2}}^{2}\right) \cap \ldots \cap\left(\mathfrak{p}_{s}^{3}+I_{l_{s}}^{2}\right)$, so that $I=\left(\mathfrak{p}_{1}^{3}+I_{l_{1}}^{2}\right) \cap J$, and $J$ corresponds to a scheme $Z^{\prime} \subset Z$ of $s-1(2,3)$-points supported on $P_{2}, \ldots, P_{s}$.

By the exact sequence $0 \rightarrow J / I \rightarrow R / I \rightarrow R / J \rightarrow 0$ and by the inductive hypothesis, we have

$$
H(Z, 3)=H\left(Z^{\prime}, 3\right)+\operatorname{dim}_{k}(J / I)_{3}=(s-1)(2 n+1)+\operatorname{dim}_{k}(J / I)_{3} .
$$

Since $H(Z, 3) \leq s(2 n+1)$, we need only prove that $\operatorname{dim}_{k}(J / I)_{3} \geq 2 n+1$, i.e., that there exist $2 n+1$ forms in $(J)_{3}$ linearly independent modulo $(I)_{3}$. By choosing appropriate coordinates we may assume that

$$
\begin{gathered}
P_{1}=[1: 0: 0: \ldots: 0: 0], \\
Q_{1}=[0: 1: 0: \ldots: 0: 0], \\
P_{2}=[0: 0: 1: 0 \ldots: 0: 0], \\
\ldots \\
P_{s}=[0: \ldots: 0: 1: 0: \ldots: 0],
\end{gathered}
$$

so that

$$
\begin{aligned}
& \mathfrak{p}_{1}=\left(y_{1}, \ldots, y_{n}\right), \quad l_{1}=\left(y_{2}, \ldots ., y_{n}\right), \\
& \mathfrak{p}_{i}=\left(y_{0}, \ldots, \hat{y}_{i}, \ldots, y_{n}\right) \text { for } 2 \leq i \leq s .
\end{aligned}
$$

Now consider the following $2 n+1$ forms:

$$
y_{0}^{3}, y_{0}^{2} y_{1},\left(y_{0}+a_{2} y_{n}\right)^{2} y_{2}, \ldots,\left(y_{0}+a_{s} y_{n}\right)^{2} y_{s}, y_{0}^{2} y_{s+1}, \ldots, y_{0}^{2} y_{n},
$$

$y_{0} y_{1}^{2},\left(y_{0}+a_{2} y_{n}\right)\left(y_{1}+b_{2} y_{n}\right) y_{2}, \ldots,\left(y_{0}+a_{s} y_{n}\right)\left(y_{1}+b_{s} y_{n}\right) y_{s}, y_{0} y_{1} y_{s+1}, \ldots, y_{0} y_{1} y_{n}$ where $a_{i}$ and $b_{i}$ are such that $\left(y_{0}+a_{i} y_{n}\right)\left(Q_{i}\right)=0,\left(y_{1}+b_{i} y_{n}\right)\left(Q_{i}\right)=0(2 \leq i \leq s)$.

For any $i, 2 \leq i \leq s$, the forms $\left(y_{0}+a_{i} y_{n}\right)^{2} y_{i}$ and $\left(y_{0}+a_{i} y_{n}\right)\left(y_{1}+b_{i} y_{n}\right) y_{i}$ are in $I_{l_{i}^{2}}$, while all the other ones are in $\mathfrak{p}_{i}^{3}$. Hence the $2 n+1$ forms above are in $J$. Passing to affine coordinates we see that they correspond (modulo $I$ ) to the following monomials in $k\left[y_{1}, \ldots, y_{n}\right]: 1, y_{1}, \ldots, y_{n}, y_{1}^{2}, y_{1} y_{2}, \ldots, y_{1} y_{s}, y_{1} y_{s+1}, \ldots, y_{1} y_{n}$ hence they are linearly independent modulo $I$.

4.2. The case $s=n$. In this case we prove the conjecture for $j \geq 4$ (which leaves the case $j=3, n=s \geq 5$ still open).

Proposition 4.3. When $j \geq 4, s=n \geq 2$, we have $\operatorname{dim} \mathcal{S}_{n, j}^{s-1}=s(2 n+1)-1$, i.e. the conjecture is true $\forall j \geq 4$, if $s=n \geq 2$.

Proof. We have to prove that $H(Z, 4)=s(2 n+1)$, hence also $H(Z, j)=s(2 n+1)$, $\forall j \geq 4$.

Since $n=s$, the points $P_{1}, \ldots, P_{s}$ will be contained in one hyperplane $H \subset \mathbb{P}^{n}$, and, by genericity, we can suppose that the lines $l_{1}, \ldots, l_{s}$ are NOT contained in $H$. Let $Z^{\prime}=\operatorname{res}_{H} Z$ be the scheme defined by the ideal $I_{Z}: I_{H}$, and let us call an " $m$-fat point" the 0 -dimensional scheme in $\mathbb{P}^{n}$ defined by the ideal $\mathfrak{p}^{m}$, where $\mathfrak{p}$ is the ideal of a point (classically, the $(m-1)^{t h}$ infinitesimal neighbourhood of the point). 
Claim. We have that:

a) $Z^{\prime}$ is given by $s$-fat points in $\mathbb{P}^{n}$, i.e. $I_{Z^{\prime}}=\mathfrak{p}_{1}^{2} \cap \ldots \cap \mathfrak{p}_{s}^{2}$;

b) $Z \cap H \subset H \cong \mathbb{P}^{n-1}$ is given by $s$-fat points in $\mathbb{P}^{n-1}$.

Proof. We can check this fact at one of the points, say $P_{1}=[1: 0: \ldots: 0]$, with $\mathfrak{q}_{1}=\left(y_{1}, y_{2}, \ldots, y_{s}\right)^{3}+\left(y_{2}, \ldots, y_{s}\right)^{2}$, and we can assume $H=\left\{y_{1}=0\right\}$. Then $\left.a\right)$ follows since the ideal of the residue is $\mathfrak{q}_{1}:\left(y_{1}\right)=\left(y_{1}, y_{2}, \ldots, y_{s}\right)^{2}$. For $\left.b\right)$, we have that the ideal of the intersection in $\frac{R}{I_{H}}$ is given by $\mathfrak{q}_{1} \cap k\left[y_{0}, y_{2}, \ldots, y_{s}\right]=\left(y_{2}, \ldots, y_{s}\right)^{2}$.

We now consider the following exact sequence of ideal sheaves:

$$
0 \rightarrow \mathcal{I}_{Z^{\prime}}(3) \rightarrow \mathcal{I}_{Z}(4) \rightarrow \mathcal{I}_{Z \cap H, H}(4) \rightarrow 0 .
$$

Recall that $H(Z, 4)=s(2 n+1)$ is equivalent to $h^{1}\left(\mathcal{I}_{Z}(4)\right)=0$, so this will be what we want to prove.

Now we can use the following powerful theorem of Alexander and Hirschowitz (see $[\underline{\mathrm{AH}}]$ ), which describes the Hilbert function for a generic set of 2 -fat points in any $\mathbb{P}^{n}$ :

Theorem AH. Let $\mathbf{X} \subset \mathbb{P}^{n}$ be a general set of 2 -fat points. Let $R=k\left[y_{0}, \ldots, y_{n}\right]$ be the coordinate ring of $\mathbb{P}^{n}$. Then

$$
H(\mathbf{X}, j)=\min \left\{s(n+1), \operatorname{dim}_{k} R_{j}\right\}
$$

except when

a) $j=2$ and $2 \leq s \leq n$;

b) $(n, j, s) \in\{(2,4,5),(3,4,9),(4,4,14),(4,3,7)\}$.

(Aside: Point $a$ ) is actually not in [AH], but was classically known (for a reference see e.g. [Ge, page 98). The exceptions in $b$ ) were known, but as statements about secant varieties of Veroneseans (see e.g. [Pa], [Te, or [RS] for other references), and it was not known that they are the only exceptions.)

So, since $\mathcal{I}_{Z^{\prime}}(3)$ and $\mathcal{I}_{Z \cap H, H}(4)$ are never in the classes of the exceptions listed above, we can use this result to show that their ideal sheaves have $h^{1}=0$ (i.e. that their Hilbert functions have reached its maximum in those degrees), as soon as $\operatorname{deg} Z^{\prime} \leq\left(\begin{array}{c}n+3 \\ 3\end{array}\right)=\operatorname{dim}_{k} R_{3}$ and $\operatorname{deg}(Z \cap H) \leq\left(\begin{array}{c}(n-1)+4 \\ 4\end{array}\right)$, respectively.

Since those two conditions are easily implied by our assumption that $n=s$, equation $(*)$ gives that $h^{1}\left(\mathcal{I}_{Z}(4)\right)=0$. That is enough to finish the proof.

4.3. The case $n<s$. For the cases where $n<s$, we can prove the following result:

Theorem 4.4. When $2 \leq n<s \leq \frac{1}{j-1}\left(\begin{array}{c}n+j-2 \\ j-2\end{array}\right)$ and $j \geq 4 \operatorname{dim} \mathcal{S}_{n, j}^{s-1}=s(2 n+1)-$ 1 , i.e. the conjecture is true also in this case.

Proof. We specialize the scheme $Z$ so that $P_{1}, \ldots, P_{s}$ lie on a hyperplane $H$ (but they are generic as points of $H$ ), while $l_{i} \notin H, \forall i$. Then, as in the previous section, $Z^{\prime}=\operatorname{res}_{H} Z$ is given by $s 2$-fat points in $\mathbb{P}^{n}$, while $Z \cap H$ is given by $s 2$-fat points in $H \cong \mathbb{P}^{n-1}$. Consider the exact sequence

$$
0 \rightarrow \mathcal{I}_{Z^{\prime}}(j-1) \rightarrow \mathcal{I}_{Z}(j) \rightarrow \mathcal{I}_{Z \cap H, H}(j) \rightarrow 0 .
$$

We want to show that, with our hypotheses, $h^{1}\left(\mathcal{I}_{Z}(j)\right)=0$. 
As soon as $(n, j, s) \notin \mathcal{A}$, with $\mathcal{A}=\{(3,4,5),(4,4,9),(5,4,14),(5,3,7)\}, Z \cap H$ is not in any of the "exceptional cases" of Theorem AH. So, assuming $(n, j, s) \notin \mathcal{A}$, in order to get that $Z \cap H$ imposes independent conditions to forms of degree $j$ in $\mathbb{P}^{n-1}$, we need only check that $\operatorname{deg}(Z \cap H)=s n \leq\left(\begin{array}{c}(n-1)+j \\ n-1\end{array}\right)$.

To show that $Z^{\prime}$ also imposes independent conditions to forms of degree $j-1$ in $\mathbb{P}^{n}$ is more delicate, since in this case the points are not generic, but contained in a hyperplane $H$. Hence the condition $(n, j, s) \notin \mathcal{A}$ is no longer sufficient. For $n=2$, the $s$-fat points lie on a line. In this case (see e.g. Gil) it is well known that in order to get $h^{1}\left(\mathcal{I}_{Z^{\prime}}(j-1)\right)=0$, we must have $j-1 \geq 2 s-1$. But $s \leq \frac{1}{j-1}\left(\begin{array}{c}n+j-2 \\ j-2\end{array}\right)=\frac{j}{2}$, hence $h^{1}\left(\mathcal{I}_{Z^{\prime}}(j-1)\right)=0$ follows.

Now let $n>2$. Consider $Z^{\prime \prime}=\operatorname{res}_{H} Z^{\prime}$ (it is given by the simple points $P_{1}, \ldots, P_{s}$ ) and $Z^{\prime} \cap H=Z \cap H$, given by the $s$-fat points in $H$. We have the exact sequence

$$
0 \rightarrow \mathcal{I}_{Z^{\prime \prime}}(j-2) \rightarrow \mathcal{I}_{Z^{\prime}}(j-1) \rightarrow \mathcal{I}_{Z^{\prime} \cap H, H}(j-1) \rightarrow 0
$$

First assume that $(n, j, s) \notin \mathcal{B}$, with $\mathcal{B}=\{(3,5,5),(4,5,9),(5,5,14),(5,4,7)\}$, otherwise $Z^{\prime} \cap H$ is one of the exceptions in Theorem AH. With this assumption we have that $H\left(Z^{\prime} \cap H, j-1\right)$ has maximum value.

In order to also have $h^{1}\left(\mathcal{I}_{Z^{\prime} \cap H}(j-1)\right)=0$, we must have (so that $Z^{\prime} \cap H$ can impose $\operatorname{deg}\left(Z^{\prime} \cap H\right)$ independent conditions in degree $\left.j-1\right)$ that $\operatorname{deg}\left(Z^{\prime} \cap H\right)=$ $s n \leq\left(\begin{array}{c}(n-1)+(j-1) \\ j-1\end{array}\right)$, i.e. $s \leq \frac{(n+j-2) \ldots(n+1)}{(j-1) !}=\frac{1}{j-1}\left(\begin{array}{c}n+j-2 \\ j-2\end{array}\right)$, which is exactly our initial condition.

What about $Z^{\prime \prime}$ ? Its Hilbert function is the same as that of $Z^{\prime \prime}$ viewed as a subscheme of $H \cong \mathbb{P}^{n-1}$ (the number of conditions imposed by $Z^{\prime \prime}$ is the same). Since $P_{1}, \ldots, P_{s}$ are generic in $H$, we will be done if $s \leq\left(\begin{array}{c}(n-1)+j-2 \\ j-2\end{array}\right)$. So, we have to show that

$$
\begin{aligned}
\left(\begin{array}{c}
(n-1)+j-2 \\
j-2
\end{array}\right) & =\frac{(n+j-3) \ldots(n+1) n}{(j-2) !} \geq \frac{1}{j-1}\left(\begin{array}{c}
n+j-2 \\
j-2
\end{array}\right) \\
& =\frac{(n+j-2) \ldots(n+1)}{(j-1) !}
\end{aligned}
$$

which, with easy computations, amounts to saying that $n \geq \frac{n+j-2}{j-1}$, which is always true.

So we have obtained $h^{1}\left(\mathcal{I}_{Z^{\prime \prime}}(j-2)\right)=0$.

Eventually, since $h^{1}\left(\mathcal{I}_{Z^{\prime \prime}}(j-2)\right)=h^{1}\left(\mathcal{I}_{Z^{\prime} \cap H}(j-1)\right)=0$, by $(\ddagger)$ we also have $h^{1}\left(\mathcal{I}_{Z^{\prime}}(j-1)\right)=0$.

Now we want $h^{1}\left(\mathcal{I}_{Z \cap H}(j)\right)=0$ too, and with this we will be done, since then we get $h^{1}\left(\mathcal{I}_{Z}(j)\right)=0$ by sequence $(\dagger)$. In order to get $h^{1}\left(\mathcal{I}_{Z \cap H}(j)\right)=0$ we must have $\operatorname{deg}(Z \cap H)=s n \leq\left(\begin{array}{c}(n-1)+j \\ n-1\end{array}\right)$, but we already checked that $\operatorname{deg}\left(Z^{\prime} \cap H\right)=s n \leq$ $\left(\begin{array}{c}n+j-2 \\ j-1\end{array}\right)=\left(\begin{array}{c}n+j-2 \\ n-1\end{array}\right)$, so this condition is satisfied and we are done.

Now we check what happens when $(n, j, s) \in \mathcal{A} \cup \mathcal{B}$, i.e. the exceptions given by Theorem AH.

Cases $(3,4,5),(4,4,9),(5,4,14),(5,3,7),(4,5,9)$ are ruled out by the condition on $s$.

Cases $(3,5,5),(5,5,14)$, and $(5,4,7)$ can be dealt with by direct computation (which we did using CoCoA), and they are not exceptions. 
4.4. More on $j=3$. Since for $j=2$ everything is known, $j=3$ is the next interesting case, offering a few other exceptions described by Conjecture 4.1. Proposition 4.2 describes what happens for $s$ small, relative to $n$. When $s$ is much bigger than $n$, something can also be said, namely:

Proposition 4.5. Let $s \geq \frac{1}{3}\left(\begin{array}{c}n+2 \\ 2\end{array}\right)+1, n \geq 2$. Then we have that $\mathcal{S}_{n, 3}^{s-1}=\mathbb{P}^{N}$.

Proof. We will prove that $h^{0}\left(\mathcal{I}_{Z}(3)\right)=0$. We specialize the points $P_{1}, \ldots, P_{s-1}$ to lie on a hyperplane $H$ so that $l_{1}, \ldots, l_{s-1}$ and $P_{s}$ are not in $H$. Then $\operatorname{res}_{H} Z=Z^{\prime}$ is given by the ideal $\mathfrak{p}_{1}^{2} \cap \ldots \cap \mathfrak{p}_{s-1}^{2} \cap \mathfrak{q}_{s}$, while $Z \cap H$ is given by $s-1$ generic 2-fat points in $H$. Consider the usual residual exact sequence

$$
0 \rightarrow \mathcal{I}_{Z^{\prime}}(2) \rightarrow \mathcal{I}_{Z}(3) \rightarrow \mathcal{I}_{Z \cap H, H}(3) \rightarrow 0 .
$$

We have, by Theorem AH, that $h^{0}\left(\mathcal{I}_{Z \cap H, H}(3)\right)=0$ as soon as $(n, s) \neq(5,8)$ and $(s-1) n \geq\left(\begin{array}{c}n+2 \\ 3\end{array}\right)$, i.e. for $s-1 \geq \frac{(n+2)(n+1)}{6}$, which is exactly our bound on $s$.

We can deal with the case $(n, s)=(5,8)$ by a direct computation (which we did using $\mathrm{CoCoA}$ ) and this gives us $h^{0}\left(\mathcal{I}_{Z}(3)\right)=0$. Hence in this particular case we are already done.

We want $h^{0}\left(\mathcal{I}_{Z^{\prime}}(2)\right)=0$. Let $X$ be the scheme defined by the ideal $\mathfrak{p}_{1}{ }^{2} \cap \ldots \cap \mathfrak{p}_{s-1}^{2}$, and $X^{\prime}=\operatorname{res}_{H} X$, i.e., $X^{\prime}$ consists of the simple points $P_{1}, \ldots, P_{s-1}$. Now consider the exact sequence

$$
0 \rightarrow \mathcal{I}_{X^{\prime}}(1) \rightarrow \mathcal{I}_{X}(2) \rightarrow \mathcal{I}_{X \cap H, H}(2) \rightarrow 0 .
$$

We have $h^{0}\left(\mathcal{I}_{X^{\prime}}(1)\right)=1$, and $h^{0}\left(\mathcal{I}_{X \cap H, H}(2)\right)=0$, as soon as $(s-1) n \geq\left(\begin{array}{c}n+1 \\ 2\end{array}\right)$, which is true by our assumption on $s$. Therefore $h^{0}\left(\mathcal{I}_{X}(2)\right)=1$. So the double hyperplane $H$, which does not contain $P_{s}$, is the only hypersurface of degree two through $X$, and hence $h^{0}\left(\mathcal{I}_{Z^{\prime}}(2)\right)=0$ follows.

4.5. The cases $s=3,4,5$. We can complete the analysis for some small values of $s$ by "brute force", i.e. checking with direct calculations those cases which are not covered by our previous results. This is of some interest because it confirms that the only exceptions are those of Conjecture 4.1 (e.g. for $s=5$ one could have expected that $\mathcal{S}_{5,3}^{4}$ "misbehaves", as $\mathcal{S}_{s, 3}^{s-1}$ does for $s=2,3,4$, which is not the case).

Proposition 4.6. For $s \leq 5$, Conjecture 4.1 is true.

Proof. For $s=2$ and $j=2$ this is already known. So, we may suppose that $j \geq 3$.

If $s=3$, then for $n \geq 4$ we are done by Proposition 4.2. When $n=3$ we have that $\mathcal{S}_{3,3}^{2}$ is one of the known exceptions, while $\operatorname{dim} \mathcal{S}_{3, j}^{2}=21$, for $j \geq 4$, by Proposition 4.3. For $n=2, \mathcal{S}_{2,3}^{2}=\mathbb{P}^{9}$, as expected. It is an easy exercise to check that there are no cubics through $Z$ in this case, so $H(Z, j)=10$ also for $j \geq 3$.

Let $s=4$; for $n \geq 5$ we are done by Proposition 4.2. When $n=4$ we have that $\mathcal{S}_{4,3}^{3}$ is one of the known exceptions, while $\operatorname{dim} \mathcal{S}_{4, j}^{3}=36$, for $j \geq 4$, by Proposition 4.3. In the cases $n=2,3$ it is an easy excercise to show that $\mathcal{S}_{3,3}^{3}, \mathcal{S}_{2,4}^{3}$ fill up the whole space $\mathbb{P}^{N}$, by Proposition 4.5 we have $\mathcal{S}_{2,3}^{3}=\mathbb{P}^{N}$, while in the other cases using CoCoA we can show that $\operatorname{dim} \mathcal{S}_{n, j}^{3}=4(2 n+1)$.

Similarly, when $s=5$, we have that Proposition 4.2 takes care of $n \geq 6$, while cases $n=5,4,3,2$ are done by CoCoA; in particular, in the case $n=5, \mathrm{CoCoA}$ shows that $\mathcal{S}_{5,3}^{4}$ is no exception. 


\section{REFERENCES}

[A] B.Adlandsvik. Joins and higher secant varieties. Math. Scand. 61 (1987), 213-222. MR 89j:14030

$[\mathrm{AH}] \quad$ J. Alexander, A. Hirschowitz, Polynomial interpolation in several variables. J. of Alg. Geom. 4 (1995), 201-222. MR 96f:14065

[CJ] M. L. Catalano-Johnson. The possible dimension of the higher secant varieties. Am. J. of Math. 118 (1996), 355-361. MR 97a:14058

[CoCoA] A. Capani, G. Niesi, L. Robbiano, CoCoA, a system for doing computations in Commutative Algebra (Available via anonymous ftp from: cocoa.dima.unige.it).

[Ge] A. V. Geramita. Inverse systems of fat points: Waring's problem, secant varieties of Veronese varieties and parameter spaces for Gorenstein ideals, Queen's Papers in Pure and Applied Math. 102 The Curves Seminar at Queen's, vol. X, Kingston, Ontario (Canada) 1999. MR 97h:13012

[Gi] A. Gimigliano. Our thin knowledge of fat points. Queens Papers in Pure and Applied Math. 83 The Curves Seminar at Queens', vol. VI, Kingston, Ontario (Canada) 1989. MR 91a:14007

[Hr] R. Harris. Algebraic Geometry, a First Course. Springer-Verlag, New York (1993). MR 93j:14001

[H] A.Hirschowitz. La methode d'Horace pour l'interpolation a plusieurs variabiles. Manuscr. Math. 50 (1985), 337-378. MR 86j:14013

[I] A.Iarrobino, Inverse systems of a symbolic algebra III: thin algebras and fat points. Compos. Math. 108 (1997), 319-356. MR 98k:13017

[IK] A. Iarrobino, V. Kanev, Power sums, Gorenstein algebras and determinantal ideals. Springer Lecture Notes in Math 1721, Springer-Verlag, New York-Berlin (1999). MR 2001d:14056

[K] V. Kanev, Chordal varietie of Veronese varieties and catalecticant matrices. Algebraic Geometry, 9. J. Math. Sci. (New York) 94 (1999), 1114-1125. MR 2001b:14078

[Pa] F. Palatini, Sulle varietà algebriche per le quali sono di dimensione minore dell'ordinario, senza riempire lo spazio ambiente, una o alcuna delle varietà formate da spazi seganti. Atti Accad. Torino Cl. Scienze Mat. Fis. Nat. 44 (1909), 362-375.

[RS] K. Ranestad, F.O. Schreyer. Varieties of sums of powers, preprint.

[Te] A. Terracini. Sulle $V_{k}$ per cui la varietà degli $S_{h}(h+1)$-seganti ha dimensione minore dell'ordinario. Rend. Circ. Mat. Palermo 31 (1911), 392-396.

[Z] F. L. Zak. Tangents and secants of algebraic varieties. Translations of Math. Monographs, vol. 127 AMS. Providence (1993). MR 94i:14053

D.I.M.E.T., Università di Genova, P.le Kennedy, 16129 Genova, Italy

E-mail address: catalisa@dima.unige.it

Department of Mathematics, Queen's University, Kingston, Ontario, Canada K7L 3N6 and Dipartimento di Matematica, Universitì di Genova, 16146 Genova, Italy

E-mail address: tony@mast.queensu.ca

E-mail address: geramita@dima.unige.it

Dipartimento di Matematica, Universitì di Bologna, 40126 Bologna, Italy

E-mail address: gimiglia@dm.unibo.it 